\title{
Artificial Intelligence in Health Care
}

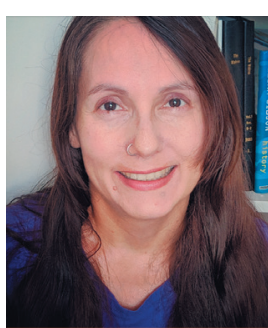

Jeannette Herrle

With a PhD in history of medicine, science and technology with over twenty years of experience in teaching and coaching, Jeanette's research interests focus on the production and dissemination of knowledge, technology, and innovation, in both healthcare and education.

I

f mobile is already entrenched as the digital technology shaping our everyday lives, Artificial Intelligence (AI) is on the threshold of being the next all-pervasive and transformative development. The automation of health care by using machines that behave "intelligently" is the major application of AI in the health sector, encompassing everything from simple pattern recognition to "smart" objects like an AI-driven insulin pump to predictive data analytics (identifying, for example, patients most at risk for hospital readmission).

$\mathrm{AI}$ is presently garnering attention and building momentum because the technology requirements are now being met. This includes computing power and storage, cloud computing, and most importantly the availability of big data sets. Add to this the ubiquitous connectivity that enables the Internet of Things (networked devices), which have the potential to act as the mechanical "body" to AI's mechanical "brain."

As in the case of mobile connectivity, the addition of "intelligence" brings a new dimension to digitization. A force for long term change in healthcare, AI's impact on both clinical practice and patient experience, whether in methods or access, is both direct-- for example, in new automated clinical tools--and indirect, through its centrality to the data-driven research that makes the emerging model of precision medicine possible.

To date, much of the expansion of AI-driven health applications has been in "smart" services/products around diagnostics: for example, smart monitors, imaging analysis, and screening tools. Imaging may represent as much as $90 \%$ of all medical data. In the news recently have been a number of screening tools using automated analysis of retinal scans, whether to diagnose age-related macular degeneration or diabetic retinopathy or identify patients at risk for heart disease.

AI tools that aid in clinical decision-making support are a related area of growth. These use the study of personal health information to inform treatment decisions for individual patients, through predictive models that anticipate how a patient will respond to a particular therapy. One relevant example is Microsoft's international collaborative eye care project (MINE), which has ongoing projects that apply machine learning to the rate of change of myopia in children or predicting outcomes of refractive surgery.

$\mathrm{AI}$ is also driving the expansion of telehealth and telehomecare. Ranging from simple bots for triage and patient education to intelligent assistants (think Amazon's Alexa) that offer homecare/caregiver support to smart homes of the near-future that incorporate continuous remote monitoring of the health of the elderly, the automation of simple tasks fills gaps in care that can occur when the demand for services--as is all but inevitable in an aging population--far outstrips the supply of care providers. 
Digital therapeutics, in which software acts as treatment modality, either via remote monitoring and or behavioural modification, is also rapidly gaining ground. A number of certified mobile apps that use gamified, remote monitoring of visual acuity (including those specifically for the management of macula diseases) are already available. These digital therapeutics have the additional advantage of making copious amounts of real-time data available on their implementation, creating feedback loops that offer the potential to optimize treatment regimens.

While there is considerable and legitimate excitement about the potential health applications of AI, the technology is still in its infancy. The base building block of AI, algorithms, underlie most of our existing technology. For example, the Fourier transform algorithm is the "recipe" that turns raw data into ultrasound or MRI images. The current excitement in AI are breakthroughs in machine learning and self-improving algorithms that can be "trained" with the input of large amounts of data on how to do a task better, like taking using vast quantities of medical images to improve the accuracy of an automated diagnosis of disease.

This kind of "narrow" AI that is good at one specific thing is here, but general AI that features all the facets of human intelligence such as planning, understanding language, recognizing objects and sounds, learning, and problem solving is still distant. Generally speaking, humans have no need to worry about being replaced by machines. While the automation of skilled labour will have an impact on some tasks traditionally the province of some healthcare specialties, patient-facing practitioners should regard them as labour-saving tools that extend their reach and help make their jobs easier, and more effective. 